

\section{Sumário}

A DISCRIMINAÇÃo RACIAL NO BRASIL E A ASCENSÃo DO POVO NEGRO: UM OLHAR A PARTIR DOS PRINCÍPIOS CONSTITUCIONAIS NA LUTA PELA CIDADANIA INCLUSIVA

Bruno Mello Correa de Barros e Rita Mara Albrecht

As MULHERES NO CONTEXTO DA SOCIEDADE DE CLASSES E SUA PARTICIPAÇÃO POLÍTICA NO PROCESSO LEGISLATIVO E EXECUTIVO DO BRASIL: RESTRIÇÕES E DESAFIOS

Rafael Bueno da Rosa Moreira e Marli Marlene Morais da Costa

FACTORES ASOCIADOS A LA VIOLENCIA DE GÉNERo EN PAREJAS ADOLESCENTES

Maria del Carmen Monreal Gimeno

Os IMPACTOS DAS TECNOLOGIAS DA INFORMAÇÃO NO FLUXO DE PESSOAS: VIOLAÇÕES DA LIBERDADE EM UM MUNDO SECURITIZADO

Elias Jacob de Menezes Neto, Jose Luis Bolzan de Morais e Victoria Layze Silva Fausto

El DELITO DE ENALTECIMIENTO TERRORISTA. ¿INSTRUMENTO DE LUCHA CONTRA EL PELIGROSO DISCURSO DEL ODIO TERRORISTA O MECANISMO REPRESOR DE REPUDIABLES MENSAJES DE RAPEROS, TWITTEROS Y TITIRITEROS?

Alfonso Galán Muñoz

As POLÍtiCAS PÚBLICAS E A PROMOÇão DA DIGNIDADE: UMA ABORDAGEM NORTEADA PELAS CApacidades (CAPABIlities APproach) propostas por Martha Nussbaum

Anna Paula Bagetti Zeifert e Janaína Machado Sturza

A INFLUÊNCIA DA MÍdia PARA O CONSUMO DE TABACO

Luís Renato Vedovato e Maria Carolina Gervásio Angelini

¿SON PARTE DEL BLOQUE DE CONSTITUCIONALIDAD LOS PRINCIPALES TRATADOS internacionales de derechos humanos de la ONU en Chile? Del texto positivo a la APLICACIÓN EN TRIBUNALES DE JUSTICIA Juan Pablo Díaz Fuenzalida

Potestad calificadora del Conservador de Bienes Raíces y Procedimiento Registral ....173 Sebastián Bozzo Hauri e Gonzalo Ruz Lartiga

DESARTICULAÇÃo INTERFEDERATIVA E CONCESSÃo DOS BENEFÍCIOS DE PRESTAÇÃo CONTINUADA (BPC) 
Aprendizagem Profissional e o poder público municipal: proposta de maior eficácia À POLÍTICA PÚBLICA

José Rodrigo Paprotzki Veloso

Direito A Conciliação Entre Trabalho E Família................................................229

Edilton Meireles de Oliveira Santos

Trabajo y diversidad funcional. La situación EN EL ORdenamiento JuRídico español 245 María Esther Carrizosa Prieto

Custos de TransaÇão como uma METAPolítica pública .........................................2276

João Luis Nogueira Matias e Ricardo José Brito Bastos Aguiar de Arruda

REVISITANDO O CONCEITO DE SERVIÇO PÚBLICO

Andre Luiz Dos Santos Nakamura

O Brasil FACE AOS NOVOS PADRÕES DE COMÉRCIO E INVESTIMENTO DOS ACORDOS INTERNACIONAIS

Michelle Sanchez Badin, Fabio Costa Morosini e David M. Trubek

Os contratos comerciais na Declaração dos Direitos de Liberdade Econômica (MP 881/19).

André Lipp Pinto Basto Lupi

O MARCO LEGAL DA INOVAÇÃO E O AUMENTO DA INTERAÇÃO ENTRE UNIVERSIDADE E EMPRESA: CONTRIBUIÇÕES PARA A CONSOLIDAÇÃO DO DIREITO FUNDAMENTAL AO DESENVOLVIMENTO ....352 Thiago Paluma e Eline Débora Teixeira

RELICITAÇÃo DAS CONCESSÕES DE GERAÇÃo DE ENERGIA ELÉTRICA E A REVERSÃo DE BENS....372 Patrícia Regina Pinheiro Sampaio e Sergio Guerra

FinANCIAMENTO TRANSGERACIONAL DA INFRAESTRUTURA VERDE FLORESTAL: O SISTEMA DE PAGAMENTO POR SERVIÇOS AMBIENTAIS COMO INSTRUMENTO DE GESTÃO DE RISCOS NA SOCIEDADE CONTEMPORÂNEA...................................................................................................390

Délton Winter de Carvalho e Kelly de Souza Barbosa

Mediação como Política Pública para Tratamento de Conflitos Consumeristas ..... 415 Fernanda Sartor Meinero e Fernando Pedro Meinero

A ParticipaÇão do AMICUS CURIAE EM DECISÕES JUdiCIAIS E SUA CONSEQUENTE CONTRIBUiÇÃo para efetivação de Políticas Públicas .429

Viviane Nobre Santana 
A ISONOMIA TRIBUTÁRIA COMO LIMITE À TRIBUTAÇÃo E À CONCESSÃO DE ISENÇÕES FISCAIS, E A INEFETIVIDADE DO CONTROLE JURISDICIONAL DESSAS ISENÇÕES..................................450

Paulo Alves da Silva Paiva e Alexandre Augusto Batista de Lima

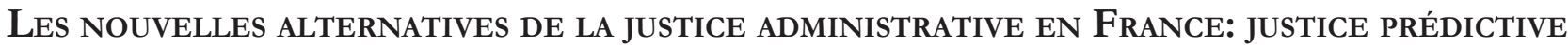
ET JUSTICE AMIABLE

Marie-Odile Diemer

O ESTADO DA LUXÚRIA: A PARÁBOLA DO BMW E A REAL DIMENSÃo DO DEBATE SOBRE A RESERVA DO POSSÍVEL: ESCASSEZ DE RECURSOS OU ORDENAÇÃo DE PRIORIDADES?................................484 Assis José Couto do Nascimento

O PODER CONSTITUINTE

José Levi Mello do Amaral Júnior

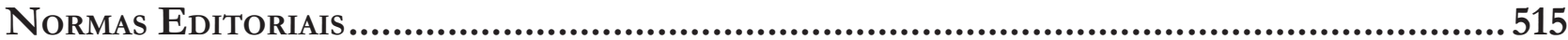

Envio dos trabalhos: 


\title{
Factores asociados a la violencia de género en parejas adolescentes*
}

\section{Factors associated with dating violence}

\author{
Maria del Carmen Monreal Gimeno**
}

\section{Resumen}

La violencia de género en parejas adolescentes o "Dating violence", hace referencia a comportamientos abusivos reiterados que un chico adolescente ejerce sobre una chica con la que mantiene o ha mantenido una relación sentimental con la intención de ejercer dominio y control sobre ella y la relación. Este comportamiento se puede manifestar de cuatro formas: agresión: física, psicológica, sexual y económica. Investigaciones recientes hacen visible una realidad que afecta a millones de mujeres de todo el mundo y permiten comprender los mecanismos de la violencia de pareja, que no se puede asimilar ni tratar como otro tipo de violencia. Uno de los hallazgos más importantes de las investigaciones más recientes es poner de manifiesto que el origen de la violencia de género se encuentra en las primeras relaciones de pareja durante adolescencia.

Las relaciones violentas que se establecen entre parejas de jóvenes adolescentes tienen múltiples causas explicativas en su raíz. Cada vez se está poniendo más de manifiesto la necesidad de prevenir este fenómeno en edades tempranas por la repercusión negativa que tendrá en el futuro de estas personas adolescentes. De ahí la necesidad de la implicación del profesorado, padres y madres en los programas de prevención y en el caso de que ésta esté instalada en la pareja en alguna de sus formas, crear programas de intervención multidisciplinar que ayudarían a los/as adolescentes que comienzan una relación de pareja violenta a salir de esta situación. Es necesaria, también, una mayor sensibilidad por parte de los medios de comunicación que presentan como normales situaciones de violencia y hay que tener presente que la violencia de género no surge de forma extrema, se va incrementando poco a poco y adquiriendo manifestaciones más alarmantes. Es necesaria pues, una educación en valores de respeto e igualdad que fomente un cambio hacia unas relaciones más igualitarias y menos discriminatorias en los roles y creencias que se establecen entre chicos y chicas cuando comienzan sus primeras relaciones de pareja.

Palabras clave: Violéncia de género, Fatores de Risco, Políticas para adolescentes; Parejas adolescentes.

género y desigualdades sociales. En: Amador, $\mathrm{L}$ y Monreal, $\mathrm{M}^{\mathrm{a}} \mathrm{C}$. (coord.). Intervención social y Género. Madrid: Narcea; Monreal Gimeno, $\mathrm{M}^{\mathrm{a}}$ C. (2010). La prevenzione della violencia di genere a traversa de l'educazione al superacento degli stereotipi. En: Campani, G. (coord.). Genere e globalizzazione. Pisa: Edizioni ETS. Email: mcmongim@gmail.com

\section{Abstract}

Gender violence in adolescent couples or "Dating violence", refers to repeated abusive behaviors that a teenage boy exercises over a girl with whom 
he maintains or has maintained a romantic relationship with the intention of exercising dominion and control over her and the relationship. This behavior can manifest itself in four ways: aggression: physical, psychological, sexual and economic. Recent research makes visible a reality that affects millions of women around the world and allows us to understand the mechanisms of intimate partner violence, which can not be assimilated or treated as another type of violence. One of the most important findings of the most recent research is to show that the origin of gender violence is found in the first couple relationships during adolescence.

The dynamics of violent relationship established between pairs of young teens seem to have explanatory multi-causal origins. The need to prevent this phenomenon at an early age is becoming increasingly apparent due to the negative impact it will have on the future of these adolescents. Hence the need for the involvement of teachers, fathers and mothers in prevention programs and in the case that it is installed in the couple in one of its forms, creating multidisciplinary intervention programs that would help adolescents who start a violent partner relationship to get out of this situation. There is also a need for greater sensitivity on the part of the media that present situations of violence as normal, and we must bear in mind that gender violence does not arise in an extreme way, it increases little by little and acquires more alarming manifestations. It is necessary, then, an education in values of respect and equality that encourages a change towards more equal and less discriminatory relationships in the roles and beliefs that are established between boys and girls when they begin their first couple relationships.

Keywords: Gender violence, risk factors, policies for teens, teenage couples.

\section{Introducción}

La investigación que analiza la violencia que se presenta en las relaciones de pareja en la adolescencia y juventud es relativamente reciente a pesar de que el abuso infantil y juvenil ha sido ampliamente estudiado con el objetivo de mejorar y prevenir el maltrato a los menores. También, le ha precedido en cuanto área de interés por parte de los investigadores/as el maltrato que tiene lugar en el contexto de una relación de pareja, especialmente la violencia que ocurre en el ámbito de las relaciones maritales ${ }^{1}$.

Es a partir de la década de los 90 cuando distintos autores/as comenzaron a señalar que la frecuencia de casos de la violencia en las relaciones de noviazgo puede ser más elevada que la marital, aunque sus consecuencias fueran menos graves o nefastas, y que la violencia se manifiesta incluso en parejas muy jóvenes². En consecuencia, en las dos últimas décadas se ha incrementado notablemente el número de investigaciones que han analizado diferentes facetas de la violencia en las parejas jóvenes como: la frecuencia de casos, las consecuencias para las víctimas, los posibles factores de riesgo y también la efectividad de programas de intervención y prevención de la violencia de género en las relaciones afectivas durante la adolescencia ${ }^{3}$. Uno de los resultados más sugerentes encontrados en estas investigaciones es que la violencia en pareja comienza, generalmente, en las primeras relaciones sentimentales durante la adolescencia, y que estos patrones violentos de comportamiento se mantienen en la etapa adulta 4 .

\footnotetext{
Alberdi, I. y Matas, N. La violencia doméstica. Informe sobre los malos tratos a mujeres en España. Barcelona: Fundación La Caixa, 2002.

2 Barnett, O.W., Miller-Perrin, C.L. y Perrin, R.D. Family violence across the lifespan: An introduction. Londres: Sage, 1997.

3 Cornelius, T.L. y Resseguie, N. Primary and secondary prevention programs for dating violence: A review of the literature. Aggression and Violent Behavior, 12, 2007, p. 364-375. Díaz-Aguado, M.J. y Carvajal, M.I. (Dirs.). Igualdad y prevención de la violencia de género en la adolescencia. Madrid: Ministerio de Igualdad y Universidad Complutense de Madrid, 2010. Muñoz-Rivas, M.J., Grana, J.L., O’Leary, D.K. y González, M.P. Aggression in adolescent dating relationships: Prevalence, justification, and health consequences. Journal of Adolescent Health, 40, 2007, p. 298-304. Ortega, R., Ortega-Rivera, J. y Sánchez, V. Violencia sexual entre compañeros y violencia en parejas adolescentes. International Journal of Psychology and Psychological Therapy, 8, 2008, p. 63-72. 4 Billingham, R.E., Bland, R. y Leary, A. Dating violence at three time periods: 1976, 1992, and 1996. Psycholycal Report, 85, 1999 ,
} 
Como ya hemos anticipado, este hecho se explica porque la agresión hacia las mujeres, no es similar a cualquier agresión, se dirige hacia ellas por el hecho de ser mujeres, como destacaron las feministas a principios de los años 70. Son resultado de unos estereotipos, que no han desaparecido del todo, según los cuales la mujer es considerada como un objeto que está al servicio del hombre por lo que éste puede imponer su voluntad incluso utilizando la fuerza. De ahí que en pequeño grado sea consentida socialmente ${ }^{5}$.

Los resultados de estas investigaciones en este ámbito constituyen una llamada de atención al destacar la importancia de la violencia en pareja en estos periodos y nos dirigen para avanzar en la dirección correcta. En consecuencia, creemos que es el momento de plantear estudios que se enmarquen dentro de modelos teóricos explicativos que examinen los mecanismos a través de los cuales se manifiesta la Violencia en parejas adolescentes. En este sentido, el propósito de este artículo es, delimitar conceptualmente la violencia de género y sus tipologías, atendiendo a las particularidades de esta etapa, analizar los factores, tanto individuales como contextuales, asociados con la Violencia en parejas adolescentes y finalmente establecer algunas conclusiones en las que se discuten los aspectos fundamentales del artículo.

\section{Consideraciones conceptuales y prevalencia de la violencia de género en parejas adolescentes (VPA)}

\subsection{Aspectos conceptuales y tipos de violencia de pareja}

La violencia de género en la pareja se define como aquel comportamiento violento de tipo físico, sexual, psicológico o económico que un hombre ejerce de forma reiterada sobre una mujer con la que mantiene o ha mantenido una relación amorosa con el objetivo de ejercer control y dominio sobre la persona y la relación. No utiliza la violencia para hacerle daño directamente, sino para conseguir que le obedezca y ejercer un control sobre ella. Se trata de una violencia instrumental, es una violencia de la que el hombre se sirve para conseguir sumisión porque cree que es lo que corresponde a las relaciones de pareja en la sociedad patriarcal. El hombre manda y la mujer obedece.

De ahí que la violencia no irrumpe de repente y con fuerza en la pareja. El hombre maltratador comienza utilizando medios sutiles como el aislamiento, la desvalorización, el control, posteriormente estos medios comienzan a ser más evidentes como los gritos, los insultos, las humillaciones, las acusaciones, las amenazas, el abuso emocional, el abuso sexual o cualquier otra estrategia de agresión eficaz para conseguir su propósito. El proceso ha sido paulatino y con algún momento de respiro de manera que la mujer víctima no es consciente de que está atrapada en una red como tela de araña, hasta que ya es muy tarde.

Como el objetivo principal del agresor es el dominio y el control de la víctima y de la relación, cuando la violencia física se presenta, normalmente el maltratador ya ha establecido un patrón previo de abuso verbal, psicológico, económico o sexual. La violencia física "sólo" se emplea si las otras formas de agresión no son eficaces. Esto implica unas creencias en las que no existe reciprocidad, la mujer no es percibida en un plano de igualdad. De ahí que se establezcan estas pautas de comportamiento que implica dominación y corrección 6 .

De acuerdo con su naturaleza existen los siguientes tipos de la violencia:

p. 574-580. Lewis, S. F. y Fremouw, W. Dating violence: A critical review of the literature. Clinical Psychology Review, 21, 2001, p. 105-127.

5 Monreal-Gimeno, M.C. Esquemas de género y violencia hacia la mujer. En Ana Ma Ruiz Tagle y Rosario Valpuesta (Eds.) Ni el aire que respiras. Pensamiento científico ante la violencia de Género. Colección Señales. Fundación Obra Social Cajasol. Sevilla, 2008.

6 Monreal-Gimeno, M.C. Esquemas de género y violencia hacia la mujer. En Ana Ma Ruiz Tagle y Rosario Valpuesta (Eds.) Ni el aire que respiras. Pensamiento científico ante la violencia de Género. Colección Señales. Fundación Obra Social Cajasol. Sevilla, 2008. 
- Física: comportamientos que van desde una bofetada hasta el extremo del asesinato. El maltrato físico, además de poner en riesgo la salud y, en los casos más extremos, la vida de las personas agredidas, provoca miedo intenso y sentimientos de humillación.

- Psicológica: comportamientos que incluyen aspectos verbales y emocionales. Aquí se incluyen actos como los insultos, los desprecios y las humillaciones. También supone conductas como ignorar (no hablar a alguien o hacer como si no existiera), chantajear y también las amenazas.

- Sexual: implica cualquier contacto sexual no deseado. Desde manosear hasta la violación.

- Económica: se refiere a conductas que implican control financiero de la víctima. Incluyen actos como prohibir o impedir que la mujer consiga una fuente propia de recursos, incluso no proveer de recursos económicos para los gastos básicos del hogar.

Durante la etapa adolescente las relaciones amorosas marcadas por la violencia física pueden incluir específicamente, empujones, golpes, patadas, bofetadas, pellizcos, tirar del cabello, estrangular o dar puñetazos. La violencia psicológica incluye comportamientos como los insultos o llamar a las chicas por apodos indeseables, tener celos con frecuencia, amenazarles con hacerles daño a ellas o amenazar suicidarse si no hacen lo que quieren, no permitir a las chicas salir con sus amistades, tratar de localizarlas continuamente (por ejemplo, a través del teléfono móvil) u ordenarles qué ropa deben vestir. Los abusos sexuales incluyen comportamientos como los manoseos y besos indeseados, relaciones sexuales obligadas, privación del uso de medios anticonceptivos o juegos sexuales por la fuerza. Por último, la violencia económica puede incluir conductas de control del gasto o animar a la chica a dejar un trabajo que le reporta ingresos económicos e independencia o incluso sus estudios.

\subsection{Algunas cifras}

Uno de los grandes problemas de la Violencia en parejas adolescentes como de toda violencia de género, es su invisibilidad y su tolerancia social (creencias generalizadas de las desigualdades de género en la sociedad patriarcal). Por una parte, los chicos y chicas no suelen sacar a la luz esta problemática, al considerarla un asunto "intimo" o justificable en algunas circunstancias, como se indica en el estudio realizado por Díaz-Aguado y Carvajal (2010) con población adolescente en el que, por ejemplo, un 35\% de los chicos adolescentes que participaron en el estudio no consideraba una conducta de violencia "controlar todo lo que hace mi pareja", frente a un $26.2 \%$ de las chicas entrevistadas. Por otra parte, hasta hace relativamente poco tiempo no se contaba con información fidedigna proveniente de una investigación rigurosa acerca de la incidencia y la prevalencia de la violencia en la pareja. Ello refleja que en el imaginario colectivo aparece una creencia que relaciona la invisibilidad con la inexistencia. Sin embargo, la realidad dista mucho de esta creencia colectiva.

En España, en los estudios con muestra adolescente española se indica que, por ejemplo, un 7.5\% de chicos y un 7.1\% de chicas admiten haber empujado o golpeado a su pareja en una o más ocasiones ${ }^{7}$. Muñoz-Rivas y sus colaboradores (2007) indican en su trabajo que aproximadamente en el 90\% de las relaciones de parejas adolescentes estudiadas existían agresiones verbales y en el 40\% agresiones físicas. En un reciente estudio, un 19\% de chicas justifican la violencia como reacción a una agresión, reduciéndose a un 5\% las chicas que admiten haber vivido situaciones de maltrato en la pareja con cierta frecuencia ${ }^{8}$. Como vemos, las cifras pueden variar considerablemente en función del tipo de instrumento de medida utilizado y la edad de los/as adolescentes pero lo cierto es que los estudios reflejan cifras muy elevadas.

\footnotetext{
González, R. y Santana, J.D. Violencia en parejas jóvenes. Análisis y prevención. Madrid: Pirámide, 2001.

8 Díaz-Aguado, M.J. y Carvajal, M.I. (Dirs.). Igualdad y prevención de la violencia de género en la adolescencia. Madrid: Ministerio de Igualdad y Universidad Complutense de Madrid, 2010.
} 
Realmente, no existe un factor que explique por sí solo por qué un adolescente se comporta de manera violenta contra su pareja y otro no lo hace. La violencia es un fenómeno sumamente complejo que hunde sus raíces en la interacción de muchos factores individuales, sociales, culturales, económicos y políticos. En consecuencia, consideramos que, para un análisis riguroso de los factores explicativos de la Violencia en parejas adolescentes es necesario abarcar una perspectiva del individuo en desarrollo (el adolescente) en permanente interacción con un ambiente social específico. A continuación, se ofrece una revisión de los factores explicativos más relevantes que se enmarcan en el modelo ecológico del desarrollo humano.

\section{El modelo ecológico aplicado al análisis de la violencia en parejas adolescentes}

En el ámbito de las Ciencias Sociales, y sobre todo en la Psicología, el modelo ecológico constituye un marco de análisis e intervención especialmente sugerente. El uso de la perspectiva ecológica tiene sus orígenes en los trabajos de Wright y Barker (1950) que toman como referentes los conceptos de espacio vital y campo psicológico de Kurt Lewin (1935). Partiendo de estos referentes, Urie Bronfenbrenner formuló el modelo ecológico del desarrollo humano que recogió en su libro "La ecología del desarrollo humano en 1987". Para este autor, la conducta es una función de la interacción de los rasgos de la persona y de sus habilidades con el ambiente $(\mathrm{C}=\mathrm{f}(\mathrm{PA})$. En otros términos, la interacción de los componentes ontogenético y de socialización produce la conducta. Esta teoría también constituye un marco de análisis de situaciones sociales $^{9}$ (Bronfenbrenner y Ceci, 1994).

En consecuencia, a diferencia de acercamientos teóricos más individuales, desde este modelo se asume una perspectiva interaccionista que posee implicaciones relevantes tanto en el análisis de las conductas como en el diseño de intervenciones. Además, desde el modelo ecológico, el individuo deja de ser un receptor pasivo, participando activamente en su entorno. En este sentido, para Bronfrenbrenner (1977) el desarrollo humano es el resultado de la acomodación entre el ser humano (activo) y los entornos inmediatos, de carácter dinámico, que le circundan.

\subsection{Contextos de desarrollo en la adolescencia desde el modelo ecológico}

Desde el modelo ecológico se considera que el ser humano se halla integrado en una tupida red de relaciones que se expresan gráficamente en estructuras concéntricas o anidadas y que representan los contextos de desarrollo o ambientes más significativos. De este modo, la conducta es el resultado de la interacción entre diferentes sistemas que se superponen y se relacionan de manera bidireccional y concéntrica; es decir, cada uno de los niveles está contenido en el siguiente. Estos contextos de desarrollo se denominan ontosistema, microsistema, mesosistema, exosistema y macrosistema. A continuación, se describen los diferentes contextos de desarrollo para la adolescencia desde el modelo ecológico:

- El ontosistema está relacionado con las características individuales del o la adolescente teniendo en cuenta las diferencias en función del género.

- Un microsistema es un patrón de actividades, roles y relaciones interpersonales que la persona en desarrollo experimenta en un entorno determinado. Normalmente, los microsistemas de una persona en desarrollo son aquellos en los que la persona actúa activamente y que influyen de una manera directa en el individuo. Ejemplos de microsistema en la adolescencia son la familia o la pandilla de amigos.

\footnotetext{
Bronfenbrenner, U. y Ceci, S.J. Nature-nature reconceptualized in developmental perspective: a bioecological model. Psychological Review, 101(4), 1994, p. 568-586.
} 
- Un mesosistema comprende las interrelaciones de dos o más entornos en los que la persona en desarrollo participa activamente. Un mesosistema es un sistema de microsistemas y, por tanto, su descripción y análisis debe realizarse en los mismos términos que los microsistemas: relaciones, actividades y roles. Por ejemplo, la coordinación de los progenitores con el profesorado para la educación de los adolescentes.

- Un exosistema se refiere a uno o más entornos que no incluyen a la persona en desarrollo como participante, pero en los cuales se producen hechos que afectan a lo que ocurre en el entorno que comprende a las personas en desarrollo. De ahí la importancia de vincular de forma efectiva los hechos que suceden en un exosistema con los hechos que acontecen en un microsistema, teniendo siempre presente los cambios evolutivos de la persona en desarrollo. Este nivel está conformado por el sistema de relaciones enmarcadas por las instituciones que median entre la cultura y el nivel familiar del o la adolescente como son la escuela, el barrio o la iglesia.

- El macrosistema se refiere a las correspondencias, en forma y contenido, de los sistemas de menor orden (micro, meso y exo) que existen o podrían existir en el nivel de la subcultura o de la cultura en su totalidad. Esta estructura anidada es el nivel más amplio e incluye las formas de organización social, las creencias culturales y los estilos de vida que prevalecen en una cultura o subcultura particular. En este nivel están incluidos las creencias patriarcales sobre las desigualdades de género basadas en los estereotipos de las que derivan los mitos del amor romántico que sostienen las relaciones de pareja poco saludables. Desde el punto de vista cultural, en las parejas adultas y sobre todo en las más jóvenes, las ideas acerca del amor y de la vida en pareja están cargadas de mitos y creencias compartidas que guían las interacciones sentimentales. Algunos de estos mitos y creencias se relacionan especialmente con la presencia de violencia en la pareja ya que se trata de prejuicios profundamente arraigados en un modelo cultural patriarcal basado en la desigualdad y asimetría de género como: "Quien bien te quiere te hará llorar “

- Por ultimo, el cronosistema, implica los cambios temporales en el ambiente que producen nuevas condiciones que inciden en el desarrollo.

Si analizamos el problema de la Violencia en parejas adolescente desde este enfoque, debemos considerar que sus causas son múltiples y complejas y que es preciso examinarlas en términos de interacción entre personas y contextos. De acuerdo con ello, es necesario analizar los principales factores tanto individuales como contextuales (relativos al individuo, la familia, la escuela, la comunidad y los medios de comunicación) asociados con los problemas de Violencia en parejas jóvenes.

\section{Factores asociados con la violencia de género en parejas adolescentes}

\subsection{Factores Individuales}

La literatura que se centra en el estudio de las consecuencias de la Violencia en parejas adolescentes señala que, además de los posibles daños físicos, la violencia hacia la pareja adolescentes está asociada con el distress psicólogico en las víctimas que es percibido por los/as adolescentes como más grave que los daños físicos ${ }^{10}$. Estudios previos también han vinculado esta violencia con la presencia de síntomas depresivos,

10 Shorey, R., Cornelius, T. y Bell, K. A critical review of theoretical frameworks for dating violence: Comparing the dating and marital fields. Aggression and Violent Behavior, 13, 2008, p. 185-194. 
ideación suicida, desórdenes alimentarios, baja autoestima y baja satisfacción con la vida, abuso de sustancias (alcohol y drogas), problemas conductuales y académicos en la escuela y un deterioro general de las condiciones físicas y mentales tanto en las víctimas como en los agresores adolescentes ${ }^{11}$. Por tanto, las relaciones de pareja violentas en la adolescencia tienen graves consecuencias psicosociales tanto para las víctimas como para los jóvenes agresores.

Un aspecto muy importante a destacar es que, el haber ejercido violencia contra una pareja anterior o tener contacto cercano con un adolescente agresor parece ser el predictor más potente de violencia en una relación futura ${ }^{12}$. En el mismo sentido, haber sido víctima de violencia por parte de su pareja o tener contacto cercano con una víctima, incrementa la posibilidad de implicarse en una nueva relación abusiva ${ }^{13}$. En general, los motivos que chicos y chicas proponen para justificar la Violencia son muy diferentes. Los chicos agreden a su pareja principalmente con objeto de dominarla, para ejercer un control sobre ella; en el caso de las chicas, por el contrario, la violencia suele ser un acto de autodefensa, un desahogo en un momento emocional de intensa ira o una respuesta ante una acción inadecuada por parte del chico (por ejemplo, una conducta de infidelidad $)^{14}$. Ahora bien, los hombres, a diferencia de las mujeres, tienden a infravalorar su propia agresión, mientras que las mujeres suelen sobrevalorar lo ocurrido y sentirse, por ello, culpables ${ }^{15}$. Estas diferencias en las reacciones posteriores a la agresión por parte de los chicos o chicas es fiel reflejo de las creencias y patrones culturales de referencia y transmitidos en el proceso de socialización.

\subsection{Factores Contextuales}

Los mitos y creencias sobre el amor romántico son estructuras cognitivas fuertemente arraigadas que configuran en buena parte las ideas sobre las relaciones de pareja que los/as adolescentes asumen como "normales". Todas ellas se enraízan profundamente en un modelo cultural basado en la desigualdad y el desequilibrio en las relaciones entre hombres y mujeres, modelo que se transmite de generación en generación mediante el proceso de socialización de género. La socialización es el proceso de transmisión de los valores, creencias, normas, actitudes y formas de conducta apropiados para la sociedad de pertenencia, de tal forma que la persona socializada asume como principios-guía de su conducta personal los objetivos socialmente valorados.

Específicamente, la socialización de género hace referencia al proceso por el cual las personas llegan a pensar y actuar de forma diferente según sean hombres o mujeres; cada mujer y cada hombre se construye mediante modelos, a través de imágenes compartidas socialmente con las que cada quien se identifica. Se espera que cada uno y cada una ejerzan el rol de género asignado de manera "adecuada". Integrarse en una cultura significa asumir los roles que la definen, no hacerlo implica romper, enfrentarse, cambiar normas fuertemente arraigadas con los consecuentes costes emocionales que esto implica ${ }^{16}$.

La violencia es una conducta que también se socializa y se aprende. De esta forma algunos autores han identificado que la mayor implicación de los chicos en formas de agresión física podría deberse al aprendi-

\footnotetext{
11 Cleveland, H., Herrera, V. y Stuewig, J. Abusive males and abused females in adolescent relationships: Risk factor similarity and dissimilarity and the role of relationship seriousness. Journal of Family Violence, 18(6), 2003, p. 325-339.

12 Vézina, J. y Hérbert, M. Risk factors for victimization in romantic relationships of young women. A review of empirical studies and implications for prevention. Trauma, Violence, and Abuse, 8(1), 2007, p. 33-66.

13 Arriaga, X.B. y Foshee, V. Adolescent dating violence: Do adolescents followin their friends', or their parents' footsteps? Journal of Interpersonal Violence, 19, 2004, p. 162-184.

${ }_{14}$ Foshee, V.A., Bauman, K.E., Linder, F., Rice, J. y Wilcher, R. Typologies of adolescent dating violence: Identifying typologies of adolescent dating violence perpetration. Journal of Interpersonal Violence, 22, 2007, p. 498-519.

15 Jackson, S.M. Issues in the dating violence research: A review of the literature. Aggression and Violent Behavior, 4, 1999 , p. 233-247.

16 Meras, A. Prevención de la violencia de género en adolescentes. En I. Silva (Coor.), Aspectos psicosociales de la violencia juvenil, no 62, 2003, pp. 143-150. 2003.
} 
zaje de "patrones de violencia diferenciados por género" ${ }^{17}$. El proceso de socialización a través del cual las personas asumen reglas y normas de comportamiento tiene lugar, fundamentalmente en el contexto de la familia. Pero debemos considerar que, en la adolescencia, existen otros microsistemas o contextos inmediatos del desarrollo tan importantes o más que la familia para los jóvenes como son los iguales o la escuela. A estos agentes socializadores fundamentales se añaden en la actualidad los medios de comunicación de masas que se ubican en el ámbito macrosocial de influencia desde los que se transmiten muchos de los mitos, creencias y representaciones del amor y la violencia.

\subsection{Los Iguales}

La relación con los iguales juega un papel fundamental en el desarrollo de la Violencia en parejas adolescentes. Se ha constatado que implicarse con grupos de amigos violentos en la escuela aumenta el riesgo de los adolescentes de ejercer violencia en las relaciones de pareja ${ }^{18}$. Mantener una estrecha vinculación con un grupo de iguales violentos en la escuela puede provocar un cierto contagio social de actitudes negativas hacia las relaciones entre hombres y mujeres y reforzar las creencias y actitudes que justifican las agresiones como aceptables y normales en las relaciones afectivas de pareja. De hecho, durante la adolescencia, la conducta violenta puede ser una forma habitual de relacionarse con los iguales y esta tendencia en el comportamiento de los adolescentes puede afectar a las relaciones afectivas y de pareja que se establecen en esta etapa ${ }^{19}$. Como se indicó anteriormente, tener contacto cercano con un adolescente agresor parece ser el predictor más potente de violencia en una relación futura ${ }^{20}$.

Además, la investigación también ha mostrado que los/as adolescentes que son víctimas de violencia escolar tienen un alto riesgo de sufrir Violencia de pareja ${ }^{21}$, ampliándose de este modo la victimización y sus consecuencias a distintas esferas de la vida de los/as adolescentes. Consideramos que esta confluencia de abusos en distintas relaciones importantes para los/as adolescentes victimizados/as configura una situación de grave riesgo que puede cursar con una baja autoestima y fuerte minusvaloración, una profunda insatisfacción con la propia vida y posible ideación suicida. Por tanto, desde todos los agentes socializadores, y, especialmente desde el ámbito educativo, es necesario facilitar pautas de detección de estas situaciones y prestar una atención especial al seguimiento de estos casos.

\subsection{La familia}

La familia es, quizás, una de las áreas que más se ha investigado cuando se trata de explicar la Violencia en parejas adolescentes. La relación de pareja que tienen padre y madre es la primera relación amorosa que perciben las niñas y niños. Foshee y sus colaboradores (2005) encontraron en su investigación una relación entre el ejercicio de Violencia en parejas adolescentes y haber presenciado o haber sido objeto de violencia en su familia de origen, independientemente del género, por la aceptación de dicha violencia y un estilo agresivo de resolución de conflictos.

Los principales factores familiares de riesgo que se relacionan con la Violencia en parejas adolescentes

\footnotetext{
17 White, J. W. Gendered aggression across the lifespan. En J. Worrell (Ed.), Encyclopedia of gender. New York: Academic Press, 2002. 18 Capaldi, D.M., Dishion, T. J., Stoolmiller, M. y Yoerger, K. Aggression toward female partners by at-risk young men: The contribution of male adolescent friendships. Developmental Psychology, 31(1), 2001, p. 61-73.

19 Muñoz-Rivas, M.J., Grana, J.L., O’Leary, D.K. y González, M.P. Aggression in adolescent dating relationships: Prevalence, justification, and health consequences. Journal of Adolescent Health, 40, 2007, p. 298-304.

20 Vézina, J. y Hérbert, M. (2007). Risk factors for victimization in romantic relationships of young women. A review of empirical studies and implications for prevention. Trauma, Violence, and Abuse, 8(1), 2007, p. 33-66.

21 Arriaga, X.B. y Foshee, V. Adolescent dating violence: Do adolescents followin their friends', or their parents' footsteps? Journal of Interpersonal Violence, 19, 2004, p. 162-184. Vézina, J. y Hérbert, M. (2007). Risk factors for victimization in romantic relationships of young women. A review of empirical studies and implications for prevention. Trauma, Violence, and Abuse, 8(1), 2007, p. 33-66.
} 
son: las prácticas parentales punitivas, la falta de cohesión afectiva, los frecuentes conflictos, los patrones inadecuados de comunicación familiar, las relaciones maritales violentas y los malos tratos y el abuso sexual de los hijos por parte de los padres, según un trabajo de revisión de artículos publicados en este ámbito desde 1986 hasta 2006 por Vézina y Hérbert (2007),

En consecuencia, el valor de estas experiencias iniciales de aprendizaje por observación es de suma importancia. A veces, se puede observar que adolescentes procedentes de hogares fríos y distantes se implican en relaciones amorosas desbordantes (controladoras, celosas, intensas) en una especie de "acción compensatoria". Sin embargo, un estilo parental que establece límites claros a los jóvenes, combinado con unas relaciones entre padres e hijos marcadas por la cercanía afectiva y la comunicación abierta y positiva parece tener una función protectora para los adolescentes ante la Violencia en perejas jóvens ${ }^{22}$.

\subsection{El profesorado}

Los Centros Educativos, tienen entre sus funciones educar para la ciudanía y una convivencia pacífica. Según Jacques Delors (1994) la educación no debe limitarse a una función instructiva sino que debe "enseñar a pensar, a hacer, a ser y a vivir con, convivir", respetando las ideas y personalidad de las otras personas. Además, el centro por sus características de convivencia y por su función educativa, se convierte en un lugar idóneo para llevar a cabo un análisis crítico de la realidad cotidiana del alumnado, así como de los mensajes educativos no formales que llegan a través de los medios de comunicación o los iguales. El objetivo de este tipo de educación debe ser transformar las bases sociales y culturales que generan en la actualidad la discriminación entre sexos. Desde este modo, dentro de los planes nacionales de erradicación de la violencia contra las mujeres, se han venido elaborando intervenciones dirigidas a prevenir la violencia de género potenciando comportamientos igualitarios en los colegios (por ejemplo, Gorrotxategi y de Haro, 1999).

$\mathrm{Al}$ igual que en el caso de los padres y madres, las profesoras/es, son personas significativas y cercanas a los adolescentes e importantes modelos de comportamiento en las relaciones inter-género y en los modos de resolución de conflictos. De ahí que el profesorado deba poner un cuidado especial en que no se premien en el aula aquellas prácticas violentas que socialmente se asocian a la masculinidad. El profesorado tiene la posibilidad de utilizar herramientas como la educación en igualdad o coeducación y el desarrollo de actividades en el aula que permitan a los jóvenes identificar y cuestionar los mitos y creencias que sustentan la Violencia en pareja.

De ahí que sea necesario que el profesorado y centros educativos en general asuman pautas igualitarias de relación y de prevención a todos los niveles: detectando y ayudando a buscar soluciones y alternativas en los casos ya establecidos, incorporando en el currículo el análisis crítico de los mitos y creencias sociales que sustentan la violencia asociada al género, y haciendo un hincapié especial en los estereotipos de género que están manejando tanto los profesores y profesoras como los chicos y las chicas.

Es cierto que la reflexión sobre los estereotipos de género junto con el debate crítico acerca de su plasmación social, puede ser una actividad educativa efectiva, aplicable en cualquier materia y más cuando se produce algún comportamiento agresivo que denota creencias que suponen desigualdad de género. Pero también lo es que una transformación significativa con respecto a la igualdad de trato sólo es posible con la implicación de todo el profesorado del centro ${ }^{23}$.

22 Vézina, J. y Hérbert, M. (2007). Risk factors for victimization in romantic relationships of young women. A review of empirical studies and implications for prevention. Trauma, Violence, and Abuse, 8(1), 2007, p. 33-66.

23 Monreal-Gimeno, M.C. Esquemas de género y violencia hacia la mujer. En Ana Ma Ruiz Tagle y Rosario Valpuesta (Eds.) Ni el aire que respiras. Pensamiento científico ante la violencia de Género. Colección Señales. Fundación Obra Social Cajasol. Sevilla, 2008. 


\subsection{Los Medios de comunicación}

Una característica esencial del mundo contemporáneo es su carácter mediático: los medios de comunicación son fácilmente accesibles e inmediatos en un mundo globalizado. Frente a otros periodos de la historia, las parejas contemporáneas ya no solo se construyen al amparo de una única exposición a modelos familiares y comunitarios cercanos sino que también se construyen bajo el importante modelado de los medios. Así, los adolescentes de hoy encuentran en la televisión, Internet y los videojuegos una fuente importante y fundamental de modelos con los que construirse una representación social del amor que guía su conducta amorosa.

Concretamente, las teleseries parecen ser un género de especial importancia en la adolescencia y muchas de ellas están pensadas especialmente para este público. Se considera que el serial televisivo es un género idóneo para el estudio de la transmisión de valores porque "representa historias cercanas a la vida cotidiana y por la función de construir modelos ya que ofrece variedad de personajes que pueden funcionar a modo de ejemplos"24. A través de estas series, los/as adolescentes ven reflejados temas sumamente relevantes para la etapa vital que atraviesan (la amistad, los romances, las relaciones con los padres), evalúan su propia situación personal y aprenden de los referentes que tienen ante sus ojos para resolver conflictos o para desenvolverse en distintas situaciones.

Pero los medios de comunicación no son neutros, satisfacen los deseos de la audiencia y transmiten los modelos tradicionales de relación, ideas acerca de las normas, estructura y conducta social (Teoría del cultivo $^{25}$ ). Además, en este proceso median otros factores y de forma importante las interacciones y estilos parentales, de tal modo que cada adolescente interpreta y percibe los contenidos televisivos. Las preferencias televisivas, si bien proceden de elecciones personales, reflejan los valores y actitudes familiares. Así, por un lado, es importante tener en cuenta el contenido que los adolescentes están viendo (qué valores, roles sociales, etc. se manejan) y, por otro lado, el contexto en el que lo están viendo, es decir la mediación que están realizando los agentes de socialización más directos (padres y profesores). Ya se ha demostrado en múltiples investigaciones el papel mediador del adulto en la visión de programas violentos en televisión y otros medios.

\section{Conclusión}

Como hemos visto a lo largo de este artículo, las cifras sobre violencia de pareja en adultos a nivel mundial son preocupantes. La visibilización de esta realidad ha fomentado el interés y la preocupación de la comunidad social, educativa y académica sobre un problema que afecta a millones de mujeres en todo el mundo. De hecho, en las últimas décadas se han desarrollado un número creciente de investigaciones que ayuda a comprender las causas y las consecuencias de este problema social y una parte de esta investigación ha centrado su interés en el origen de la violencia en las primeras relaciones de pareja que ocurre durante adolescencia.

Las dinámicas de relaciones violentas que se establecen entre parejas de jóvenes adolescentes parecen tener raíces explicativas multicausales. De esta forma, analizar este problema desde el marco teórico explicativo del modelo ecológico de los factores asociados con la violencia de género en parejas adolescentes, implica estudiar los diferentes factores individuales, familiares, escolares y sociales que nos permitan comprenderlo de una forma multidisciplinar y sistémica. La implicación de padres y madres, profesorado y de la comunidad en

24 Montero, Y. Televisión, valores y adolescencia. Barcelona: Gedisa, 2006, p. 26

25 Gerbner, G., Gross, L., Morgan, M. y Signorielli, N. Growing up with television: The cultivation perspective. En J. Bryant y D. Zillmann (Eds.), Media effects (pp. 17-41). Hillsdale, NJ: Erlbaum, 1994. 
los programas de prevención y la intervención multidisciplinar en todo el proceso ayudaría a los/as adolescentes que comienzan una relación de pareja violenta a salir de ella. Por otra parte, una mayor sensibilidad de los medios de comunicación hacia esta realidad fomentaría un cambio hacia una mayor igualdad y no discriminación en los roles, creencias y mitos que se establecen entre chicos y chicas cuando comienzan sus primeras relaciones de pareja.

Por último, cada vez son más las voces que desde todos los ámbitos, sobre todo desde el judicial, llaman la atención sobre la importancia de cuidar el proceso educativo y de formación de los/as jóvenes para erradicar esa terrible lacra social que constituye la violencia de género.

\section{Referencias}

Alberdi, I. y Matas, N. (2002). La violencia doméstica. Informe sobre los malos tratos a mujeres en España. Barcelona: Fundación La Caixa.

Arriaga, X.B. y Foshee, V. (2004). Adolescent dating violence: Do adolescents followin their friends', or their parents' footsteps? Journal of Interpersonal Violence, 19, 162-184.

Barnett, O.W., Miller-Perrin, C.L. y Perrin, R.D. (1997). Family violence across the lifespan: An introduction. Londres: Sage.

Billingham, R.E., Bland, R. y Leary, A. (1999). Dating violence at three time periods: 1976, 1992, and 1996. Psycholycal Report, 85, 574-580.

Bronfenbrenner, U. (1977). Toward an experimental ecology of human development. American Psychologist, 32, 513-531.

Bronfenbrenner, U. (1979/1987). The ecology of human development. Cambridge, Mass: Harvard University Press. (Ed. cast.: La ecología del desarrollo humano. Barcelona: Paidós).

Bronfenbrenner, U. y Ceci, S.J. (1994). Nature-nature reconceptualized in developmental perspective: a bioecological model. Psychological Review, 101(4) 568-586.

Capaldi, D.M., Dishion, T. J., Stoolmiller, M. y Yoerger, K. (2001). Aggression toward female partners by atrisk young men: The contribution of male adolescent friendships. Developmental Psychology, 31(1), 61-73.

Cleveland, H., Herrera, V. y Stuewig, J. (2003). Abusive males and abused females in adolescent relationships: Risk factor similarity and dissimilarity and the role of relationship seriousness. Journal of Family Violence, 18(6), 325-339.

Cornelius, T.L. y Resseguie, N. (2007). Primary and secondary prevention programs for dating violence: A review of the literature. Aggression and Violent Behavior, 12, 364-375.

Delors, Jacques (1994). “Los cuatro pilares de la educación”, en La Educación encierra un tesoro. México: El Correo de la UNESCO, pp. 91-103.

Díaz-Aguado, M.J. y Carvajal, M.I. (Dirs.) (2010). Igualdad y prevención de la violencia de género en la adolescencia. Madrid: Ministerio de Igualdad y Universidad Complutense de Madrid.

Foshee, V.A., Bauman, K.E., Linder, F., Rice, J. y Wilcher, R. (2007). Typologies of adolescent dating violence: Identifying typologies of adolescent dating violence perpetration. Journal of Interpersonal Violence, 22, 498-519. 
Foshee, V.A., Ennett, S.T., Bauman, K. E., Benefield, T. y Suchindran, C. (2005). The association between family violence and adolescent dating violence onset. Does it vary by race, socioeconomic status, and family structure? The Journal of Early Adolescence, 23, 317-344.

Gerbner, G., Gross, L., Morgan, M. y Signorielli, N. (1994). Growing up with television: The cultivation perspective. En J. Bryant y D. Zillmann (Eds.), Media effects (pp. 17-41). Hillsdale, NJ: Erlbaum.

González, R. y Santana, J.D. (2001). Violencia en parejas jóvenes. Análisis y prevención. Madrid: Pirámide.

Gorrotxategi, M. y de Haro, I.M. (1999). Materiales didácticos para la prevención de la violencia. Málaga: Consejería de Educación y Ciencia. Junta de Andalucía.

Jackson, S.M. (1999). Issues in the dating violence research: A review of the literature. Aggression and Violent Behavior, 4, 233-247.

Lewin, K. (1935). A dynamic theory of personality. New York: McGraw-Hill.

Lewis, S. F. y Fremouw, W. (2001). Dating violence: A critical review of the literature. Clinical Psychology Review, 21, 105-127.

Meras, A. (2003). Prevención de la violencia de género en adolescentes. En I. Silva (Coor.), Aspectos psicosociales de la violencia juvenil, no 62 (pp. 143-150). Madrid: Instituto de la Juventud.

Monreal-Gimeno, M.C. (2008). Esquemas de género y violencia hacia la mujer. En Ana Ma Ruiz Tagle y Rosario Valpuesta (Eds.) Ni el aire que respiras. Pensamiento científico ante la violencia de Género. Colección Señales. Fundación Obra Social Cajasol. Sevilla

Montero, Y. (2006). Televisión, valores y adolescencia. Barcelona: Gedisa.

Muñoz-Rivas, M.J., Grana, J.L., O’Leary, D.K. y González, M.P. (2007). Aggression in adolescent dating relationships: Prevalence, justification, and health consequences. Journal of Adolescent Health, 40, 298-304.

Ortega, R., Ortega-Rivera, J. y Sánchez, V. (2008). Violencia sexual entre compañeros y violencia en parejas adolescentes. International Journal of Psychology and Psychological Therapy, 8, 63-72.

Shorey, R., Cornelius, T. y Bell, K. (2008). A critical review of theoretical frameworks for dating violence: Comparing the dating and marital fields. Aggression and Violent Behavior, 13, 185-194.

Vézina, J. y Hérbert, M. (2007). Risk factors for victimization in romantic relationships of young women. A review of empirical studies and implications for prevention. Trauma, Violence, and Abuse, 8(1), 33-66.

White, J. W. (2002). Gendered aggression across the lifespan. En J. Worrell (Ed.), Encyclopedia of gender. New York: Academic Press.

Wright, H.F. y Barker, R.G. (1950). Methods in psychological ecology. Lawrence: Department of Psychology, University of Kansas. 
Para publicar na revista Brasileira de Políticas Públicas, acesse o endereço eletrônico www.rbpp.uniceub.br

Observe as normas de publicação, para facilitar e agilizar o trabalho de edição. 